\title{
Best Possible Region of Attraction for a Class of Unstable Systems Using Short Horizon MPC Concepts
}

\author{
Adrian M. Medioli, Maria M. Seron, and Richard H. Middleton \\ ARC Centre for Complex Dynamic Systems and Control \\ School of Electrical Engineering and Computer Science \\ The University of Newcastle, NSW 2308 Australia \\ Email: amedioli@ee.newcastle.edu.au
}

\begin{abstract}
This paper proposes a control strategy for input constrained second order systems with one unstable mode. For this class of systems, the largest region of attraction under bounded control is bounded in one direction whilst infinite in the other. Standard MPC type control with guaranteed stability either requires large control horizons or typically yields a much smaller region of attraction. We show that by extending MPC concepts a control strategy with a horizon of one is developed that provides asymptotic stability with the largest possible region of attraction under bounded control.
\end{abstract}

\section{INTRODUCTION}

With bounded control, unstable systems by their very nature have limits on the set of states that can be taken to the origin. This set of states forms what is often referred to as the controllable region of the system. Knowledge of these limits has been used in other research [1][2][3] to design controllers that expand the region of attraction to the controllable region itself. None of this research is directly connected with Model Predictive Control (MPC).

MPC is a popular control strategy due to its inherent ability to handle constraints. However, stability of MPC can not be guaranteed in the presence of constraints and with a finite horizon, without additional conditions being placed on the formulation. These additional conditions in general result in conservative regions of attraction that are highly dependent on the horizon length [4][5]. As a consequence there is a trade-off between a low computational burden associated with short horizon MPC, and the size of the region of attraction. Extending the region of attraction to match that of the controllable region using MPC would require an infinite horizon. For second order systems having one real unstable pole, this paper presents a novel saturation strategy, motivated by horizon-one MPC, where the saturation depends both on the input constraints and the system's unstable state. This strategy is shown to achieve a region of attraction equal to the controllable region.

\section{SYSTEM}

We consider a class of open loop unstable, linear, time invariant, second order systems with the following state space representation,

$$
\dot{x}(t)=A x(t)+B u(t)
$$

where $x(t)=\left[\begin{array}{ll}x_{1}(t) & x_{2}(t)\end{array}\right]^{T}$ is the state vector in $\mathbb{R}^{2}$, $u(t)$ is the input in $\mathbb{R}$ and $(A, B)$ is controllable.
We assume that the matrices in (1) have the following form,

$$
A=\left[\begin{array}{cc}
a_{11} & 0 \\
0 & a_{22}
\end{array}\right], B=\left[\begin{array}{l}
b_{1} \\
b_{2}
\end{array}\right]
$$

where $a_{11}>0$ and $a_{22}<0$, indicating that $x_{1}$ is the unstable state and $x_{2}$ is the stable state.

Further we will assume that the input magnitude is constrained, that is $u_{\min } \leq u \leq u_{\max }$ with $u_{\max }>0, u_{\min }<0$ and without loss of generality that $b_{1}>0$ and $b_{2}>0$.

\section{CONTROL}

In this section observations on the limitations of any possible controller and a discussion on the use of MPC are presented.

\section{A. Controllable Region for Unstable Constrained Systems}

The controllable region for an input constrained system is the set of states that are controllable by an input that satisfies the required bounds [1]. For a system with a single unstable state the controllable region is determined by the values of the unstable state that can be cancelled in finite time by the maximum and minimum available control magnitudes.

Thus, for our system, the controllable region denoted by $R_{\max }$ is given by [6] as,

$$
R_{\text {max }} \triangleq\left\{x \in \mathbb{R}^{2}: \frac{-b_{1} u_{\max }}{a_{11}}<x_{1}<\frac{-b_{1} u_{\min }}{a_{11}}\right\} \text {. }
$$

\section{B. Model Predictive Control}

MPC is a strategy that computes the control action at each time via the minimisation of a function of the system's future inputs and states over a "prediction horizon". Input (and state) constraints can be directly included in the optimisation [7]. Without additional precautions, MPC does not inherently guarantee stability for every feasible solution of the optimisation unless the prediction horizon is of infinite length. However, optimisation over an infinite horizon is intractable in the presence of constraints. With a finite horizon, asymptotic stability can be guaranteed under certain conditions if the predicted state at the end of the horizon lies in a "terminal set" satisfying certain properties [4]. Under these conditions, MPC is guaranteed to be asymptotically stabilising for any feasible state, that is, for any state for which there exists a control sequence that satisfies the required bounds and can steer the state to the terminal set at the end of the horizon. 
From the previous discussion we can see that the region of attraction for MPC with guaranteed stability is directly dependent on the length of the horizon. In order to obtain a large region of attraction the horizon may need to be large, hence affecting the complexity of implementation.

Our aim is to achieve a region of attraction equal to the controllable region. Thus, new conditions for stability as discussed below have to be considered.

\section{Limitation Aware MPC}

For the class of systems described in Section II, MPC with a finite horizon and only input constraints does not guarantee stability from all initial states in the controllable region. Given the discussion in Section III-A, it would seem natural to add a constraint on the unstable state that will keep the system in the controllable region. This idea motivates the control strategy proposed in Section IV.

\section{Optimal Control From MPC CONCEPTS}

In this section we present a controller that can be shown to be the limit, under infinitely fast sampling, of a discrete-time MPC controller with horizon one.

For a given state weighting $Q=Q^{T}>0$ let $P$ be the positive definite solution to the continuous time algebraic Riccati equation, $A^{T} P+P A-P B B^{T} P+Q=0$. Define the differential cost function ${ }^{1}$,

$$
J(u)=u^{T} u+x^{T} Q x+\frac{d}{d t}\left(x^{T} P x\right)
$$

with optimal unconstrained control, $u^{*}=-B^{T} P x$. Then with (1) and $u^{*}$ we have,

$$
J(u)=\left(u-u^{*}\right)^{T}\left(u-u^{*}\right) .
$$

Therefore, the 'MPC' type control with unit horizon is,

$$
u_{m}=\underset{u_{\min } \leq u \leq u_{\max }}{\arg \min } J(u)
$$

which is satisfied by the control law,

$$
u_{m}=\operatorname{sat}(-K x)
$$

where $K=B^{T} P$ is a linear gain matrix and sat() is the standard saturation function. We observe, for future reference, that the gain matrix $K=\left[\begin{array}{ll}k_{1} & k_{2}\end{array}\right]$ is stabilising for (1) and hence it satisfies,

$$
\begin{aligned}
a_{11} a_{22}-a_{11} k_{2} b_{2}-a_{22} k_{1} b_{1} & >0 \\
b_{1} k_{1}+b_{2} k_{2}-a_{11}-a_{22} & >0 .
\end{aligned}
$$

It can be checked that (8), (9) imply, $k_{1}>0$.

A constraint on the unstable state can be handled by introducing additional components to the cost function (4),

$$
J_{\delta}(u)=J(u)+J_{\max }(u)+J_{\min }(u)
$$

where

$$
\begin{aligned}
J_{\text {max }}(u) & =\frac{1}{2 \delta} \frac{d}{d t}\left[\max \left\{x_{1}-x_{1 \text { max }}, 0\right\}\right]^{2} \\
& =\left\{\begin{array}{cl}
0 & \text { if } x_{1} \leq x_{1 \text { max }} \\
\frac{1}{\delta} \dot{x}_{1}\left(x_{1}-x_{1 \text { max }}\right) & \text { if } x_{1}>x_{1 \text { max }}
\end{array}\right.
\end{aligned}
$$

${ }^{1}$ In the sequel, the dependence on time of all variables is omitted.

$$
\begin{aligned}
J_{\min }(u) & =\frac{1}{2 \delta} \frac{d}{d t}\left[\min \left\{x_{1}-x_{1 \min }, 0\right\}\right]^{2} \\
& =\left\{\begin{array}{cl}
0 & \text { if } x_{1} \geq x_{1 \min } \\
\frac{1}{\delta} \dot{x}_{1}\left(x_{1}-x_{1 \min }\right) & \text { if } x_{1}<x_{1 \min }
\end{array}\right. \\
& x_{1 \min }=-b_{1} u_{\max } / a_{11}+\varepsilon \\
& x_{1 \max }=-b_{1} u_{\min } / a_{11}-\varepsilon
\end{aligned}
$$

and

$$
\varepsilon<\min \left\{-b_{1} u_{\min } / a_{11}, b_{1} u_{\max } / a_{11}\right\} .
$$

Minimising $J_{\delta}(u)$ under input constraints and taking the limit as $\delta$ approaches zero produces the following control law.

$$
\begin{aligned}
u_{m}^{\prime} & =\lim _{\delta \rightarrow 0} \underset{u_{\min } \leq u \leq u_{\max }}{\arg \min } J_{\delta}(u) \\
& = \begin{cases}u_{\max } & \text { if } x_{1}<x_{1 \min } \\
\operatorname{sat}(-K x) & \text { if } x_{1 \min } \leq x_{1} \leq x_{1 \max } \\
u_{\min } & \text { if } x_{1}>x_{1 \max }\end{cases}
\end{aligned}
$$

This new control law implies that for $x_{1 \min } \leq x_{1} \leq x_{1 \max }$ the state space is partitioned into three control regions. A linear control region $u_{\min } \leq-K x \leq u_{\max }$, where the linear control $u=-K x$ is applied; and two input constrained regions $-K x<u_{\min }$ and $-K x>u_{\max }$, corresponding to $u=u_{\min }$ and $u=u_{\max }$, respectively.

In addition for $x_{1}>x_{1 \max }$ or $x_{1}<x_{1 \min }$ the resulting input is such that $x_{1}$ moves back toward the boundary at the maximum rate given the available input range.

On the lines $x_{1}=x_{1 \max }$ and $x_{1}=x_{1 \min }$ the right hand side of the differential equations describing the closed loop system (1),(14) are discontinuous over a range of $x_{2}$. For this kind of differential equations, existence and uniqueness of solutions on the discontinuous portions of these lines may be considered using differential inclusions theory as proposed by Filippov [8].

In general discontinuities occur on a switching surface $s(x)=0$ and existence and uniqueness of solutions on this surface in the sense of Filippov can be guaranteed if all points satisfy the following conditions [9].

$$
\lambda_{+}\left(x^{*}\right)<0 \text { or } \lambda_{-}\left(x^{*}\right)>0
$$

where

$$
\begin{aligned}
& \lambda_{+}\left(x^{*}\right)=\lim _{x \rightarrow x^{*}} \lambda_{+}(x) ; \lambda_{-}\left(x^{*}\right)=\lim _{x \rightarrow x^{*}} \lambda_{-}(x) ; \\
& x^{*} \in\{x: s(x)=0\}
\end{aligned}
$$

and

$$
\begin{aligned}
& \lambda_{+}(x)=\frac{d s}{d t}=\frac{\partial s}{\partial x} \dot{x} \text { for } s>0 \\
& \lambda_{-}(x)=\frac{d s}{d t}=\frac{\partial s}{\partial x} \dot{x} \text { for } s<0 .
\end{aligned}
$$

We consider the case $x_{1}=x_{1 \max }$, the switching surface is given by $s(x)=x_{1}-x_{1 \max }=0$. By using the control law (14), $\lambda_{+}(x)$ and $\lambda_{-}(x)$ become,

$$
\begin{aligned}
& \lambda_{+}(x)=\dot{x}_{1}=a_{11} x_{1}+b_{1} u_{m i n} \text { for } s>0 \\
& \lambda_{-}(x)=\dot{x}_{1}=a_{11} x_{1}+b_{1} \operatorname{sat}(-K x) \text { for } s<0 .
\end{aligned}
$$


First we consider $\lambda_{+}\left(x^{*}\right)$,

$$
\lambda_{+}\left(x^{*}\right)=a_{11} x_{1 \max }+b_{1} u_{\min }=-\varepsilon a_{11} \text { [using (12)]. }
$$

Since $a_{11}>0, \varepsilon>0$, we have,

$$
\lambda_{+}\left(x^{*}\right)=-\varepsilon a_{11}<0 .
$$

Hence the first condition in (15) is always true, guaranteeing existence and uniqueness of the solution of (1), (14) in the sense of Filippov, along the switching surface.

Let us now consider $\lambda_{-}\left(x^{*}\right)$ for the case $k_{2}>0$. Using (19) we have,

$$
\lambda_{-}\left(x^{*}\right)=\left\{\begin{array}{lll}
a_{11} x_{1 \max }+ & \\
b_{1} \operatorname{sat}\left(-K x^{*}\right) & >0 & \text { if } x_{2}^{*}<x_{2 s} \\
a_{11} x_{1 \max }+ & & \\
b_{1} \operatorname{sat}\left(-K x^{*}\right) & \leq 0 & \text { if } x_{2 s} \leq x_{2}^{*}<x_{2 c} \\
-\varepsilon a_{11} & <0 & \text { if } x_{2}^{*} \geq x_{2 c}
\end{array}\right.
$$

where

$$
\begin{aligned}
& x_{2 c}=u_{\text {min }}\left(b_{1} k_{1}-a_{11}\right) / a_{11} k_{2}+\varepsilon k_{1} / k_{2} \\
& x_{2 s}=u_{\text {min }}\left(b_{1} k_{1}-a_{11}\right) / a_{11} k_{2}+\varepsilon\left(b_{1} k_{1}-a_{11}\right) /\left(b_{1} k_{2}\right) .
\end{aligned}
$$

Conditions (20) and (21) imply that for the portion $x_{2}^{*}<$ $x_{2 s}$ the trajectories on both sides of the switching surface $x_{1}=x_{1 \text { max }}$, point toward it. For the portion $x_{2}^{*} \geq x_{2 s}$ the trajectories on the right of the switching surface point toward it with those on the left pointing away. In addition, for $x_{2 s} \leq x_{2}^{*}<x_{2 c}$, the right hand side of (1), (14) is discontinuous on the switching surface whereas for $x_{2}^{*} \geq x_{2 c}$ it is continuous. The trajectories for all cases are illustrated in Fig. 1.

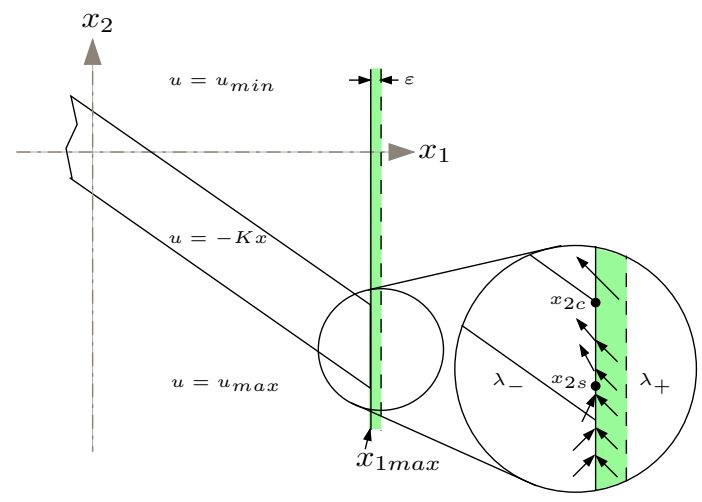

Fig. 1. Depiction of control action on the switching surface

Hence, for $x_{2}^{*}<x_{2 s}$, the trajectories slide along the switching surface $x_{1}=x_{1 \max }$ [9]. For $x_{2 s} \leq x_{2}^{*}<x_{2 c}$, it may be shown that the trajectory defined in the sense of Filippov has only $x^{*}$ in common with the switching surface and goes from right to left through $x^{*}$ [9]. For $x^{*} \geq x_{2 c}$ the differential equations (1), (14) have a unique solution in the standard sense and the resulting trajectories cross the switching surface from right to left.

Further it can be shown that if $k_{2}<0$ the analysis of the switching surface trajectories remains the same, only the definition of the portions are changed.
Similar arguments can be made for the case $x_{1}=x_{1 \text { min }}$.

Using this approach the resulting continuous time closed loop system is a piecewise affine system similar to a one step horizon MPC formulation of the problem.

Note that (14) coincides with the linear controller $u=$ $-K x$ near the origin and hence asymptotic (exponential) stability is automatically ensured in a region around the origin. The following sections demonstrate that (14) actually achieves asymptotic stability with a region of attraction equal to the controllable region.

\section{DEFINITIONS}

In this section we give the definition of various critical elements for the analysis presented in subsequent sections.

\section{A. Critical Points}

One of the features that dominates the behaviour of the system are the equilibrium points that are active within each region of the piecewise affine closed loop system. The linear control region $u_{\min } \leq-K x \leq u_{\max }$ has a stable equilibrium point at the origin, and the input constrained regions corresponding to $u=u_{\max }$ and $u=u_{\min }$ have the equilibrium points defined below.

Definition 1 (Saturated Equilibrium Points): The equilibrium points for the minimum and maximum inputs denoted as $x_{e+}$ and $x_{e-}$ respectively, are given by,

$$
\begin{aligned}
& A x_{e+}+B u_{\min }=0 \\
& A x_{e-}+B u_{\max }=0 .
\end{aligned}
$$

Using (2), we obtain,

$$
\begin{aligned}
& x_{e+}=\left[\begin{array}{l}
x_{1 e+} \\
x_{2 e+}
\end{array}\right]=\left[\begin{array}{l}
-b_{1} u_{\min } / a_{11} \\
-b_{2} u_{\min } / a_{22}
\end{array}\right] \\
& x_{e-}=\left[\begin{array}{l}
x_{1 e-} \\
x_{2 e-}
\end{array}\right]=\left[\begin{array}{l}
-b_{1} u_{\max } / a_{11} \\
-b_{2} u_{\max } / a_{22}
\end{array}\right] .
\end{aligned}
$$

To further aid the stability analysis it is important to determine at which points along the boundary lines $u_{\max / \min }=$ $-K x$ do the state trajectories have derivatives parallel to these lines or back into the linear control region $u_{\text {min }} \leq$ $-K x \leq u_{\max }$. These points help define the boundary of an invariant sub-region of the linear control region, making them significant for our analysis.

\section{Definition 2 (Boundary Tangential Trajectory Points):}

The boundary tangential trajectory point corresponding to $u_{\max }=-K x$ is denoted by $x_{T \max }$ and is determined by the following equations,

$$
\left[\begin{array}{c}
K A \\
-K
\end{array}\right] x_{\max }=\left[\begin{array}{c}
-K B u_{\max } \\
u_{\max }
\end{array}\right]
$$

where $K=\left[\begin{array}{ll}k_{1} & k_{2}\end{array}\right]$ is the linear control gain matrix in (14). After some manipulation using (2) this results in the following explicit expression for $x_{T \max }=$ $\left[\begin{array}{ll}x_{1 T \max } & x_{2 T \max }\end{array}\right]^{T}$,

$$
\begin{aligned}
& x_{1 \text { Tmax }}=-u_{\max }\left(a_{22}-K B\right) /\left(k_{1}\left(a_{22}-a_{11}\right)\right) \\
& x_{2 \text { Tmax }}=u_{\max }\left(a_{11}-K B\right) /\left(k_{2}\left(a_{22}-a_{11}\right)\right)
\end{aligned}
$$


By symmetry a similar expression is obtained for the boundary tangential trajectory point on the line $u_{\min }=-K x$ and is given by $x_{T \min }=\left[\begin{array}{ll}x_{1 T \min } & x_{2 T \min }\end{array}\right]^{T}$,

$$
\begin{aligned}
& x_{1 T \text { min }}=-u_{\text {min }}\left(a_{22}-K B\right) /\left(k_{1}\left(a_{22}-a_{11}\right)\right) \\
& x_{2 \text { Tmin }}=u_{\text {min }}\left(a_{11}-K B\right) /\left(k_{2}\left(a_{22}-a_{11}\right)\right) .
\end{aligned}
$$

To simplify the equations in the sequel we will define two new variables $x_{T+}$ and $x_{T-}$, that always represent the tangential points with positive and negative $x_{2}$ components. That is, $x_{T+}=\left[\begin{array}{ll}x_{1 \max } & x_{2 T+}\end{array}\right]^{T}$ and $x_{T-}=$ $\left[\begin{array}{ll}x_{1 T \text { min }} & x_{2 T-}\end{array}\right]^{T}$, where

$$
\begin{aligned}
& x_{2 T+}= \begin{cases}x_{2 T \max } & \text { if } k_{2}>0 \\
x_{2 T \min } & \text { if } k_{2}<0\end{cases} \\
& x_{2 T-}=\left\{\begin{array}{l}
x_{2 T \min } \text { if } k_{2}>0 \\
x_{2 T \max } \text { if } k_{2}<0
\end{array} .\right.
\end{aligned}
$$

Hence, if $x_{2 T-} \leq x_{2} \leq x_{2 T+}$, the state trajectories on the lines $-K x=u_{\min / \max }$ have derivatives directed toward the unconstrained region $u_{\min } \leq-K x \leq u_{\max }$.

To define the analysis regions in the next section we also require that the following properties hold,

$$
\begin{aligned}
& x_{1 \text { Tmin }} \leq x_{1 e+}-\varepsilon \\
& x_{1 \text { Tmax }} \geq x_{1 e-}+\varepsilon .
\end{aligned}
$$

If the gain $K$ is stabilising, there always exists an $\varepsilon$ such that (33), (34), hold, as we show next. We begin by considering (33). From (29) and (25) we have,

$$
x_{1 T \text { min }}-x_{1 e+}=\frac{-u_{\text {min }}\left(a_{11} a_{22}-a_{11} k_{2} b_{2}-a_{22} k_{1} b_{1}\right)}{a_{11} k_{1}\left(a_{22}-a_{11}\right)} .
$$

By the system description we have $u_{\min }<0, a_{11}>0$, $a_{22}-a_{11}<0$ and by the stabilising property of $K$ we have $a_{11} a_{22}-a_{11} k_{2} b_{2}-a_{22} k_{1} b_{1}>0$ and $k_{1}>0$ (see (8) and (9)). Thus $x_{1 T \text { min }}-x_{1 e+}<0$ and we can always choose $\varepsilon$ sufficiently small to satisfy (33). A similar analysis can be performed for (34).

We note that if $\varepsilon$ satisfies (33), (34) then (13) holds.

\section{B. Analysis Regions}

Definition 3 (Region $R_{\max -\varepsilon}$ ): The region $R_{\max -\varepsilon}$ is defined as

$$
R_{\max -\varepsilon} \triangleq\left\{x \in \mathbb{R}^{2}: x_{1 \min } \leq x_{1} \leq x_{1 \max }\right\}
$$

where $x_{1 \min }$ and $x_{1 \max }$ are as in (11), (12), with $\varepsilon$ satisfying (33), (34), (Fig. 2).

Note that the boundaries of $R_{\max -\varepsilon}$ are within $\varepsilon$ of those of the controllable region (3).

Definition 4 (Region $R_{2 T}$ ): The region $R_{2 T}$ is defined as,

$$
R_{2 T} \triangleq\left\{x \in R_{\max -\varepsilon}: x_{2 T-} \leq x_{2} \leq x_{2 T+}\right\}
$$

where $x_{2 T-}$ and $x_{2 T+}$ are the boundary tangential trajectory points defined in (31), (32), (Fig. 2).

Definition $5\left(R_{\operatorname{lin} 2 T}\right)$ : The region $R_{\operatorname{lin} 2 T}$ is defined as,

$$
\begin{array}{r}
R_{l i n 2 T} \triangleq\left\{x \in \mathbb{R}^{2}: u_{\min } \leq-K x \leq u_{\max }\right. \\
\text { and } \left.x_{2 T-} \leq x_{2} \leq x_{2 T+}\right\}
\end{array}
$$

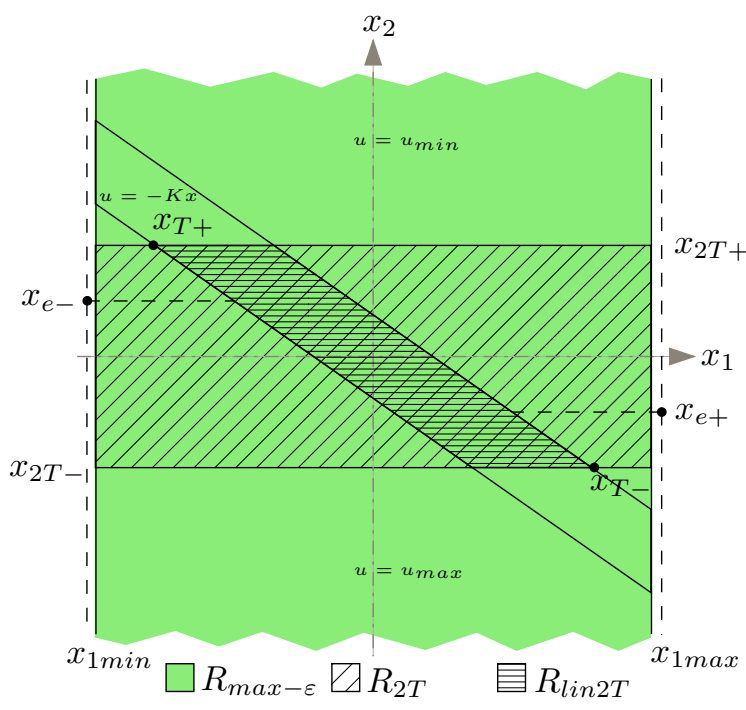

Fig. 2. Depiction of analysis regions

where $x_{2 T-}$ and $x_{2 T+}$ are defined in (31), (32), (Fig. 2). $\quad \nabla$ In the sequel we require the following assumption,

Assumption 1:

a) $x_{2 T+}>x_{2 e-}$

b) $x_{2 T-}<x_{2 e+}$

For the case where $k_{2}>0$, it can be shown that conditions a) and b) are equivalent to (8), hence the assumption automatically holds if $K$ is stabilising.

However, if $k_{2}<0$ the assumption holds if and only if,

$$
u_{\text {min }} a_{22}\left(a_{11}-K B\right)+u_{\max } k_{2} b_{2}\left(a_{22}-a_{11}\right)<0 \text {. }
$$

\section{Best Possible Region of Attraction}

In this section we show that the controller (14) asymptotically stabilises system (1) achieving a region of attraction equal to the controllable region.

The results are presented in a series of lemmas that provide the construction elements for the proof of the final theorem.

Lemma 1: The regions $R_{\operatorname{lin} 2 T}, R_{2 T}, R_{\max -\varepsilon}$ and $R_{\max }$ are nested.

Proof: By (3) and Definition 3 it follows that $R_{\max -\varepsilon} \subset R_{\max }$. Similarly, by Definitions 4 we have that $R_{2 T} \subset R_{\max -\varepsilon}$.

By Definitions 4 and 5, region $R_{\operatorname{lin} 2 T}$ shares the upper and lower boundaries with $R_{2 T}$ and the lateral boundaries of $R_{l i n 2 T}$ defined by $-K x=u_{\max / \min }$ intersect the upper and lower boundaries at $x_{T+}$ and $x_{T-}$, respectively (See Fig. 2). These points are within the lines $x_{1}=x_{1 \max }$ and $x_{1}=x_{1 \min }$ since $\varepsilon$ was chosen to satisfy (33) and (34). Hence, $R_{\operatorname{lin} 2 T}$ is a subset of $R_{2 T}$. The result then follows.

Lemma 2: For the system defined in Section II the following properties hold,

a) $\dot{x}_{2} \leq a_{22}\left(x_{2 T+}-x_{2 e-}\right)$ for all $x$ such that $x_{2} \geq x_{2 T+}$

b) $\dot{x}_{2} \geq a_{22}\left(x_{2 e+}-x_{2 T-}\right)$ for all $x$ such that $x_{2} \leq x_{2 T-}$ Proof:

a) The stable state satisfies, $\dot{x}_{2}=a_{22} x_{2}+b_{2} u$ where $u_{\min } \leq u \leq u_{\max }$. From (23), the equilibrium point 
for $u=u_{\max }$ satisfies, $a_{22} x_{2 e-}+b_{2} u_{\max }=0$. Then, for $x_{2} \geq x_{2 T+}$ and since $b_{2}>0, a_{22}<0$, we have,

$$
\begin{aligned}
\dot{x}_{2} & =a_{22}\left(x_{2}-x_{2 e-}\right)+a_{22} x_{2 e-}+b_{2} u \\
& \leq a_{22}\left(x_{2}-x_{2 e-}\right)+\underbrace{a_{22} x_{2 e-}+b_{2} u_{\max }}_{0} \\
& \leq \underbrace{a_{22}\left(x_{2}-x_{2 T+}\right)}_{\leq 0}+a_{22}\left(x_{2 T+}-x_{2 e-}\right) \\
\dot{x}_{2} & \leq a_{22}\left(x_{2 T+}-x_{2 e-}\right) .
\end{aligned}
$$

b) Similarly, using the equation for the equilibrium point $u=u_{\min }$ and the facts that $b_{2}>0, a_{22}<0$, we have that $\dot{x}_{2} \geq a_{22}\left(x_{2 e+}-x_{2 T-}\right)$ when $x_{2} \leq x_{2 T-}$.

Lemma 3: For the system defined in Section II, the following properties hold.

a) For all $x$ such that $x_{1} \leq x_{1 \max }$ and with saturated input $u=u_{\min }$ then $\dot{x}_{1} \leq-a_{11} \varepsilon$,

b) For all $x$ such that $x_{1} \geq x_{1 \text { min }}$ and with saturated input $u=u_{\max }$ then $\dot{x}_{1} \geq a_{11} \varepsilon$.

\section{Proof:}

a) For $u=u_{\text {min }}$, the unstable state satisfies, $\dot{x}_{1}=$ $a_{11} x_{1}+b_{1} u_{\text {min }}$. From (22), the equilibrium point for $u=u_{\min }$ satisfies, $a_{11} x_{1 e+}+b_{1} u_{\min }=0$. Then, for $x_{1} \leq x_{1 \max }$ and since $b_{1}>0, a_{11}>0$ and $x_{1 \max }=x_{1 e+}-\varepsilon$ we have,

$$
\begin{aligned}
\dot{x}_{1} & =a_{11}\left(x_{1}-x_{1 \max }\right)+a_{11} x_{1 \max }+b_{1} u_{\min } \\
& =\underbrace{a_{11}\left(x_{1}-x_{1 \max }\right)}_{\leq 0}+\underbrace{a_{11} x_{1 e+}+b_{1} u_{\min }}_{0}-a_{11} \varepsilon \\
\dot{x}_{1} & \leq-a_{11} \varepsilon .
\end{aligned}
$$

b) Similarly for $x_{1} \geq x_{1 \min }$ and $u=u_{\max }$ we have, $\dot{x}_{1} \geq a_{11} \varepsilon$.

Lemma 4: For the closed loop system (1), (14), in the region outside of $R_{\max -\varepsilon}$ but inside $R_{\max }$, the following properties hold.

a) All trajectories with initial state $x$ such that $x_{1 \max }<$ $x_{1}<x_{1 e+}$ reach $x_{1}=x_{1 \max }$ in finite time.

b) All trajectories with initial state $x$ such that $x_{1 e-}<$ $x_{1}<x_{1 \min }$ reach $x_{1}=x_{1 \text { min }}$ in finite time.

Proof:

a) From (14), $u=u_{\text {min }}$ everywhere in this region, hence the following applies.

$$
\begin{aligned}
\dot{x}_{1} & =a_{11} x_{1}+b_{1} u_{\text {min }} \\
& =a_{11}\left(x_{1}-x_{1 e+}\right)+\underbrace{a_{11} x_{1 e+}+b_{1} u_{m i n}}_{=0}
\end{aligned}
$$

Let $\chi^{+}=x_{1}-x_{1 e+}$, then $\dot{\chi}^{+}=a_{11} \chi^{+}$and $\chi^{+}(t)=$ $e^{a_{11} t} \chi^{+}(0)$. By definition $\chi^{+}(0)<0$, implying that,

$$
\chi^{+}(t)=-\varepsilon \text { for some finite } t .
$$

and hence $x_{1}(t)$ reaches $x_{1 \max }$ in finite time. b) Similarly for $x_{1 e-}<x_{1}<x_{1 \min }, u=u_{\max }$ and letting $\chi^{-}=\left(x_{1}-x_{1 e-}\right)$ we have,

$$
\chi^{-}(t)=\varepsilon \text { for some finite } t
$$

and hence $x_{1}(t)$ reaches $x_{1 \min }$ in finite time.

Lemmas 5 to 7 below show (forward) invariance of the analysis regions.

Lemma 5: For the closed loop system (1), (14), the region $R_{\max -\varepsilon}$ is invariant.

Proof: From the analysis in Section IV, the control law (14) ensures that, $x_{1}(t) \leq x_{1 \min }$ and $x_{1}(t) \geq x_{1 \max }$, provided the initial state is in $R_{\max -\varepsilon}$. Hence, all trajectories starting in $R_{\max -\varepsilon}$ remain in $R_{\max -\varepsilon}$, proving its invariance.

Lemma 6: For the closed loop system (1), (14), and under Assumption 1 the region $R_{2 T}$ is invariant.

Proof: Considering the boundary of this region the following statements can be proved. Using Assumption 1 then from Lemma 2 all trajectories starting on $x_{2}=x_{2 T+}$ have the property $\dot{x}_{2} \leq a_{22}\left(x_{2 T+}-x_{2 e-}\right)<0$. Similarly, all trajectories starting on $x_{2}=x_{2 T}$ - have the property $\dot{x}_{2} \geq$ $a_{22}\left(x_{2 e+}-x_{2 T-}\right)>0$. From (14) it can be checked that $u=u_{\max }$ on the left boundary of $R_{2 T}$ and $u=u_{\min }$ on its right boundary. Then, from Lemma 3 all trajectories starting on $x_{1}=x_{1 \max }$ have the property $\dot{x}_{1} \leq-a_{11} \varepsilon<0$ and all trajectories starting on $x_{1}=x_{1 \text { min }}$ have the property $\dot{x}_{1} \geq$ $a_{11} \varepsilon>0$. These four properties show that all trajectories on the boundary of $R_{2 T}$ are directed into $R_{2 T}$ and hence the region is invariant.

Lemma 7: For the closed loop system (1), (14), and under Assumption 1 the region $R_{\operatorname{lin} 2 T}$ is invariant and the origin is asymptotically stable with a region of attraction that includes $R_{\text {lin } 2 T}$.

Proof: From Lemma 2 and Assumption 1 the upper and lower bounds of $R_{\operatorname{lin} 2 T}$ have trajectories directed into the region and by Definition 2 all trajectories on the boundaries given by $-K x=u_{\max / \min }$ and $x_{2 T-} \leq x_{2} \leq x_{2 T+}$ are also directed into the region. Hence the region $R_{l i n 2 T}$ is invariant. Within the region $R_{l i n 2 T}$ the control law is unconstrained and given by the linear feedback $u=-K x$. Hence the origin is asymptotically stable and its region of attraction includes $R_{\operatorname{lin} 2 T}$.

The following theorem presents the main result of this paper.

Theorem 1: For the closed loop system (1), (14), and under Assumption 1 the origin is asymptotically stable with a region of attraction $R_{\max }$.

Proof: First consider points $x$ outside of the interior of $R_{\max -\varepsilon}$ but inside $R_{\max }$, that is $\left\{x: x \in R_{\max }\right.$ and $x \notin$ $\left.\operatorname{int} R_{\max -\varepsilon}\right\}$. By Lemma 4, all trajectories starting in this set reach $R_{\max -\varepsilon}$ in finite time. Next consider points $x$ outside the interior of $R_{2 T}$ but inside $R_{\max -\varepsilon}$, that is,

$$
\mathbb{X}_{O 2 T} \triangleq\left\{x: x \in R_{\max -\varepsilon} \text { and } x \notin \operatorname{int} R_{2 T}\right\} .
$$

By Lemma 2 and Assumption 1,

$\left\{x \in \mathbb{X}_{O 2 T}: x_{2} \geq x_{2 T+}\right\} \Longrightarrow \dot{x}_{2} \leq a_{22}\left(x_{2 T+}-x_{2 e-}\right)<0$

$\left\{x \in \mathbb{X}_{O 2 T}: x_{2} \leq x_{2 T-}\right\} \Longrightarrow \dot{x}_{2} \geq a_{22}\left(x_{2 e+}-x_{2 T-}\right)>0$. 
All trajectories in the direction of $x_{2}$ move toward the $R_{2 T}$ boundary and since $R_{\max -\varepsilon}$ is invariant by Lemma 5, all states $x \in \mathbb{X}_{O 2 T}$ must reach region $R_{2 T}$ in finite time.

Now consider all points within $R_{2 T}$ but outside the interior of $R_{\operatorname{lin} 2 T}$ that is,

$$
\mathbb{X}_{\text {Olin } 2 T} \triangleq\left\{x: x \in R_{2 T} \text { and } x \notin \operatorname{int} R_{\text {lin } 2 T}\right\} .
$$

By Lemma 3,

$$
\begin{aligned}
& \left\{x \in \mathbb{X}_{\text {Olin } 2 T}:-K x \geq u_{\text {max }}\right\} \Longrightarrow \dot{x}_{1} \geq a_{11} \varepsilon>0 \\
& \left\{x \in \mathbb{X}_{\text {Olin } 2 T}:-K x \leq u_{\min }\right\} \Longrightarrow \dot{x}_{1} \leq-a_{11} \varepsilon<0 .
\end{aligned}
$$

All trajectories in the direction of $x_{1}$ move toward the $R_{l i n 2 T}$ boundary and by Lemma $6 R_{2 T}$ is invariant. Then all states $x \in \mathbb{X}_{\text {Olin } 2 T}$ must reach $R_{\text {lin } 2 T}$ in finite time.

By Lemma $7 R_{\operatorname{lin} 2 T}$ is invariant and the origin is asymptotically stable. Therefore all trajectories starting in $R_{\max }$ converge asymptotically to the origin, proving the result.

\section{EXAMPLE}

To show the implications of Theorem 1 and to illustrate the important results for this class of systems, an example is provided.

The system chosen for the example has the following data,

$$
A=\left[\begin{array}{cc}
1 & 0 \\
0 & -1
\end{array}\right] ; B=\left[\begin{array}{l}
1 \\
1
\end{array}\right] ; \begin{aligned}
& u_{\max }=1 ; \\
& u_{\min }=-1
\end{aligned}
$$

The equilibrium points for this sample system are, $x_{e+}=$ $\left[\begin{array}{cc}1 & -1\end{array}\right]^{T}$ and $x_{e-}=\left[\begin{array}{ll}-1 & 1\end{array}\right]^{T}$. The controllable region of this system is $R_{\max }=\left\{-1<x_{1}<1\right\}$. The gain $K$ in (14) is selected as $K=\left[\begin{array}{ll}2.4 & 0.4\end{array}\right]$.

Fig. 3 shows the linear control region (denoted as $R_{\text {lin }}$ ), the region of attraction with guaranteed stability for MPC with horizon 15 (denoted as $R_{\mathrm{MPC} 15}$ ) and the trajectories for various control strategies. Also shown is $\mathcal{O}_{\infty}$ [10], the maximal invariant set for the system $\dot{x}=(A-B K) x$ inside the region $u_{\min } \leq-K x \leq u_{\max }$. This set is used as the terminal set in the MPC strategy with guaranteed stability. The solid line shows the trajectory of the continuous

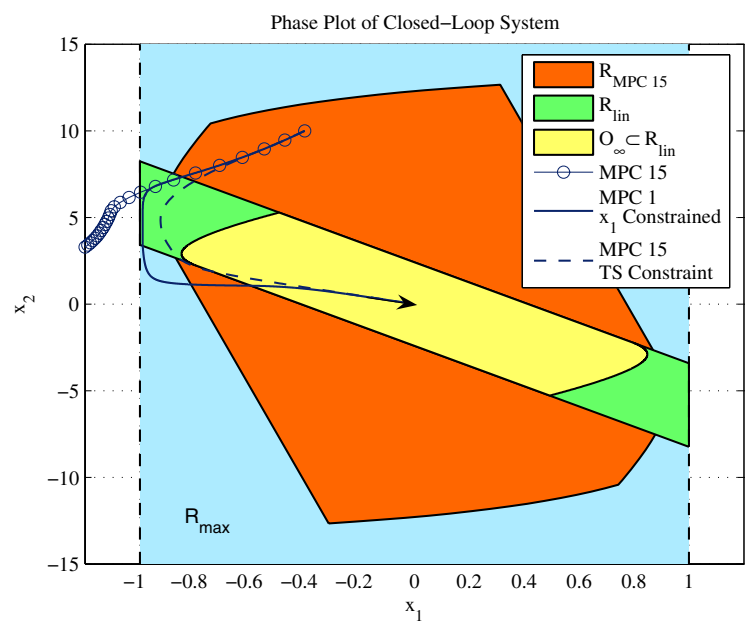

Fig. 3. Plot of relevant regions and trajectories using example system time closed loop system (1), (37), (14), resulting from our proposed strategy formulated with input and unstable state constraints. The solid line marked with circles, showing the trajectory that tracks outside the controllable region is produced by an MPC formulation without the unstable state constraint and horizon 15 . The dashed line is the trajectory resulting from an MPC formulation with horizon 15 without the unstable state constraint but with stability guaranteed by using $\mathcal{O}_{\infty}$ as a terminal constraint set (see Section III-B).

This example shows that to achieve guaranteed stability using MPC concepts without the unstable state constraint requires a relatively long horizon, and introduces infeasible results dependent on the horizon length (i.e. initial states outside $R_{\text {MPC15 }}$ are infeasible for the MPC problem). By introducing the unstable state constraint, stabilisation is achieved even with a horizon of one, and the entire controllable region $R_{\max }$ is both the feasible region and the region of attraction for this control.

\section{CONCLUSION}

This paper has presented a control strategy for a class of second order systems with one unstable and one stable mode. By applying MPC type control with the constraint such that the unstable mode remains within the controllable region of the system, it has been shown that the entire controllable region becomes the region of attraction for the origin.

Further research is being conducted to extend the presented concepts to higher order systems, and to show that this theory is applicable to discrete time implementations.

\section{REFERENCES}

[1] A. Teel, "Anti-windup for exponentially unstable linear systems," International Journal of Robust and Nonlinear Control, vol. 9, no. 10, pp. 701-716, 1999.

[2] J.-Y. Favez, P. Mullhaupt, B. Srinivasan, D. Bovin, and J. Lister, "Enhancing tokomak control given power supply voltage saturation," ICALEPCS2003, pp. 40-42, 2003.

[3] E. Schuster, M. Walker, D. Humphreys, and M. Krstic, "Plasma vertical stabilization in the presence of coil voltage saturation in the DIII-D Tokamak," in Proceedings of the June 4-6 2003 American Control Conference, Denver, Colorado, 2003.

[4] D. Mayne, J. Rawlings, C. Rao, and P. Scokaert, "Constrained model predictive control: Stability and optimality," Automatica, vol. 36, pp. 789-814, 2000.

[5] A. Casavola, M. Gianelli, and E. Mosca, "Global predictive regulation of null-controllable input-saturated linear systems," IEEE Transactions on Automatic Control, vol. 44, no. 11, pp. 2226-2230, November 1999.

[6] L. Scibile and B. Kouvaritakis, "Stability region for a class of openloop unstable linear systems: theory and application," Automatica, vol. 36, pp. 37-44, 2000.

[7] J. Rawlings, "Tutorial: Model predictive control technology," in Proceedings of the June 1999 American Control Conference, San Diego, California, 1998.

[8] A. Filippov, "Differential equations with discontinuous right hand sides," Translations of the American Mathematical Society, vol. 62, 1960.

[9] S. Sastry, Nonlinear Systems. Analysis, Stability and Control. Springer, 1999.

[10] E. Gilbert and K. Tan, "Linear systems with state and control constraints: the theory and application of maximal output admissible sets," IEEE, vol. 36, pp. 1008-1020, 1991. 